\title{
Teeth and physical fitness in a community-dwelling 40 to 79-year-old Japanese population
}

This article was published in the following Dove Press journal:

Clinical Interventions in Aging

29 June 2016

Number of times this article has been viewed

\author{
Akinari Inui' \\ Ippei Takahashi² \\ Kaori Sawada ${ }^{2}$ \\ Akimoto Naoki ${ }^{2}$ \\ Toshirou Oyama' \\ Yoshihiro Tamura' \\ Toshiyuki Osanai ${ }^{1}$ \\ Anna Satake' \\ Shigeyuki Nakaji² \\ Wataru Kobayashi' \\ 'Department of Oral and Maxillofacial \\ Surgery, Hirosaki University Graduate \\ School of Medicine, ${ }^{2}$ Department of \\ Social Medicine, Hirosaki University \\ Graduate School of Medicine, \\ Hirosaki City, Aomori Prefecture, \\ Japan
}

Purpose: Decline in the number of teeth and physical fitness begins from 40 years of age; however, several epidemiological studies have identified relationships between oral conditions and physical performance parameters in community-dwelling elderly population. The aim of this study was to validate the relationship between the muscle mass and its function and oral conditions (number of teeth and dental occlusion) after 40 years of age in a community-dwelling population in Japan.

Materials and methods: The subjects comprised of 552 volunteers (198 males and 354 females, 40-79 years) who participated in the Iwaki Health Promotion Project in 2013. Multiple linear regression analyses were performed with the measures of the muscle mass and its function as objective variables and the measures of the number of teeth, age, body mass index, medical history, serum albumin concentration, smoking status, habitual alcohol intake, marital status, education levels, and exercising habits as explanatory variables. The relationships between the Eichner index and the muscle mass and its function were analyzed using analysis of covariance, with adjustment for confounding factors.

Results: After adjusting for confounding factors, the number of teeth was shown to be an independent risk factor for the timed $10 \mathrm{~m}$ walk test (in females) and the skeletal muscle mass of the whole body (in males). The results also revealed that the timed $10 \mathrm{~m}$ walk test was significantly correlated with the Eichner index (Classes A and C in females were correlated).

Conclusion: This cross-sectional study on a Japanese community-dwelling population revealed relationships between oral conditions and the muscle mass and its function. However, the interpretation of our results was hampered by a lack of data, including those on socioeconomic status and longitudinal observations. Future research exploring teeth loss and the muscle mass and its function is warranted.

Keywords: number of teeth, sarcopenia, timed $10 \mathrm{~m}$ walk test, hand grip strength, skeletal muscle mass

\section{Introduction}

A major change associated with human aging is a progressive decline in skeletal muscle mass (SMM), which is a downward spiral that may lead to decreased strength and function. Age-related decline in physical fitness is related to many problems in the elderly population. According to previous epidemiological studies on the elderly, physical performance may be related to maintenance of physical fitness, improvement of life motivation, quality of life (QOL), and nutrition. ${ }^{1-4}$ Österberg et al suggested that a lack of occlusal support was related to general physical muscle weakness. ${ }^{5}$ This study was based on three 70 -year-old cohorts $(n=1,380)$. Furthermore, Yamaga et al reported that dental occlusion is associated with dynamic strength of the lower extremities, agility, and balance in the elderly. ${ }^{6}$ This sample consisted of 591 individuals aged 70 years and
Department of Oral and Maxillofacial Surgery, Hirosaki University Graduate School of Medicine, 5 Zaifutyo, Hirosaki, 036-8562 Aomori Prefecture, Japan $\mathrm{Tel}+8 \mathbf{I} \quad \mathbf{7 7} 395 \quad 27$

Fax $+81 \quad 177395128$

Email hI4gm308@hirosaki-u.ac.jp 
158 aged 80 years selected from the registry of residents in Niigata City, Japan. Participants of previous epidemiological studies have tended to be $>70$ years old. Moreover, walking speed is very important in QOL; however, there have been no reports on the relationship between walking speed and oral condition. Sarcopenia is a syndrome characterized by progressive and generalized loss of SMM and strength, with a risk of adverse outcomes such as physical disability, poor QOL, and death. ${ }^{7,8}$ The European Working Group on Sarcopenia in Older People (EWGSOP) recommends using the presence of both low muscle mass and muscle function (strength or performance) for the diagnosis of sarcopenia. The age-dependent loss of SMM begins at $\sim 40$ years of age and becomes prominent after 50 years of age in Japanese adults. ${ }^{9}$ Some studies have suggested that an exercise habit in middle-aged people was a protective factor against sarcopenia in older age and is effective for maintaining muscle strength and physical performance in older age. ${ }^{10,11}$ From the National Dental Survey, the prevalence of periodontitis in those aged 40-45 and 55-59 years was $25.6 \%$ and $47.0 \%$, respectively. ${ }^{12}$ Decline in the number of teeth and the muscle mass and its function begins from 40 years of age; however, several epidemiological studies have identified relationships between oral conditions and physical performance parameters in community-dwelling older adults. However, few reports have studied the relationship between the number of teeth and the muscle mass and its function related to diagnosis of sarcopenia after the age of 40 years. Therefore, the aim of this study was to validate the relationship between muscle mass and its function (timed $10 \mathrm{~m}$ walk test, hand grip strength, and SMM of the whole body) and oral function (the number of teeth and dental occlusion) after 40 years of age in a community-dwelling population in Japan after adjusting for explanatory variables such as age, body mass index (BMI), diabetes, hypertension, cardiovascular disease, stroke, serum albumin concentration (g/dL), smoking status, habitual alcohol intake, marital status, level of education, and exercise habits.

\section{Materials and methods}

\section{Participants}

The subjects comprised of 607 volunteers (222 males and 385 females, all $\geq 40$ years) who participated in the Iwaki Health Promotion Project in 2013. Exclusion criteria for our study were medical history of cancer, stroke, and ischemic heart disease. Subjects with incomplete data were excluded from the study. Eventually, 552 participants (198 males and 354 females) were included in our study. This study was approved by the Ethics Committee of Hirosaki University School of Medicine, and all subjects had provided written informed consent before participating in the study. The demographic data (age, sex, education level, and marital status), lifestyle (smoking and drinking), and medical history were obtained from self-report questionnaires and interviews. For exercise habit, the participants were instructed to choose from the following five items: none, 1 day/week, 2-3 days/week, 4-5 days/week, 6 days/week, or every day. The height and weight of the subjects were measured, and their BMI was calculated using the following equation: BMI $\left(\mathrm{kg} / \mathrm{m}^{2}\right)=$ weight $(\mathrm{kg}) /($ height $[\mathrm{m}] \times$ height $[\mathrm{m}])$. In previous studies, serum albumin concentration $(\mathrm{g} / \mathrm{dL})$ was considered for confounding factor because it represents nourishment. ${ }^{2,6}$ Nonfasting blood samples were obtained, and plasma concentrations of albumin were measured using standard assay procedures in conjunction with a completely automated system (SRL Ltd., Tokyo, Japan).

\section{Oral examination}

Dental examinations were conducted by trained and experienced dentists under artificial lighting, with both the dentist and the subject in seated position. The teeth present were defined as healthy, carious, or treated. According to the records of dental examinations, the subjects were divided into three classes based on the Eichner index, which was used as an indicator of occlusal condition. ${ }^{13}$ The Eichner index is based on existing natural tooth contact between the maxilla and the mandible in the bilateral premolar and molar regions (presence of tooth contact was defined as the presence of a natural tooth on the maxilla and corresponding mandible, including wisdom teeth, but excluding the remaining roots or root caps). Class A represents the contact in all four support zones. Class B represents the contact in one to three zones or in the frontal region only. Finally, Class $\mathrm{C}$ represents the absence of tooth contact.

\section{Timed $10 \mathrm{~m}$ walk test}

Each subject was instructed to walk at a comfortable, normal pace for $16 \mathrm{~m}$ measured. ${ }^{14}$ However, only the $10 \mathrm{~m}$ in between were timed using a stopwatch to eliminate the effects of acceleration and deceleration. ${ }^{15,16}$ The starting and stopping of the performance time coincided with the toes of the leading foot crossing the 3 and $13 \mathrm{~m}$ mark, respectively.

\section{Hand grip strength}

Maximum hand grip strength was measured in both the dominant and nondominant hands using a digital dynamometer (TKK5401 Grip-D; Takei Scientific Instruments Company Limited, Niigata, Japan). The score was selected as the best of the trials for both grip strengths. 


\section{SMM of the whole body}

Measurements of the SMM of the whole body $(\mathrm{kg})$ were obtained from the bioelectrical impedance analysis of each participant using a Tanita MC-190EM body composition analyzer (Tanita Corporation, Tokyo, Japan), which uses an eight-point tactile electrode impedance meter. Participants were instructed to stand upright on the electrodes and grasp the handles of the analyzer, and then to slightly abduct their arms and remain still during the measurement. The participant's SMM $(\mathrm{kg})$ was computed through proprietary algorithms and was displayed on the analyzer's control panel and recorded. The body weight $(\mathrm{kg})$ of the participants was automatically determined when they stood on the electrodes of the scale platform.

\section{Statistical analysis}

Spearman's correlation coefficient was applied when testing the association between two continuous variables. The Kruskal-Wallis test was used to analyze continuous variable distribution on categorical variable categories. Multiple linear regression analyses were performed with the measures of muscle mass and its function as objective variables and the measures of the number of teeth, age, BMI, diabetic and hypertensive readings, serum albumin concentration (g/dL), smoking status, habitual alcohol intake, marital status (living vs not living with a spouse), level of education, and exercising habits as explanatory variables. The relationship between the Eichner index and the muscle mass and its function was analyzed using analysis of covariance, after adjusting for explanatory variables. The level of statistical significance was set at 0.05 . SPSS for Windows (v22; IBM Corporation, Armonk, NY, USA) was applied for the statistical analysis.

\section{Results}

\section{Sample characteristics}

The subjects were divided into two groups according to sex. The clinical characteristics of the subjects are shown in Table 1 . Timed $10 \mathrm{~m}$ walk test, hand grip strength, SMM, and BMI were greater in males compared with females. Diabetes mellitus, smoking status, habitual alcohol intake, and marital status were significantly different between males and females.

\section{Relationship between number of remaining teeth and physical fitness}

Table 2 presents the results of the multiple linear regression analyses between the number of remaining teeth and the muscle mass and its functions. After adjusting for
Table I Demographic characteristics of the subjects

\begin{tabular}{|c|c|c|c|}
\hline \multirow[t]{2}{*}{ Variable } & \multirow{2}{*}{$\frac{\text { Males }}{(n=198)}$} & \multirow{2}{*}{$\frac{\text { Females }}{(n=354)}$} & \multirow[t]{2}{*}{$P$-value } \\
\hline & & & \\
\hline Number of remaining teeth & $22.4 \pm 8.4$ & $21.5 \pm 8.7$ & $0.267^{\mathrm{a}}$ \\
\hline \multicolumn{4}{|l|}{ Eichner index } \\
\hline Class A & 149 (75.3\%) & $248(70.1 \%)$ & $0.272^{b}$ \\
\hline Class B & $28(14.1 \%)$ & $52(14.7 \%)$ & \\
\hline Class C & $21(10.6 \%)$ & $54(15.3 \%)$ & \\
\hline Timed $10 \mathrm{~m}$ walk test $(\mathrm{s})$ & $4.4 \pm 0.9$ & $4.7 \pm 0.9$ & $<0.00 \mathrm{I}^{\mathrm{a}}$ \\
\hline Hand grip strength (kg) & $41.0 \pm 7.6$ & $25.5 \pm 4.2$ & $<0.00 I^{\mathrm{a}}$ \\
\hline SMM (kg) & $49.3 \pm 5.4$ & $35.3 \pm 3.0$ & $<0.00 \mathrm{I}^{\mathrm{a}}$ \\
\hline Age (years) & $59.9 \pm 10.1$ & $59.8 \pm 9.8$ & $0.932^{\mathrm{a}}$ \\
\hline BMI $\left(\mathrm{kg} / \mathrm{m}^{2}\right)$ & $23.5 \pm 2.8$ & $22.5 \pm 3.2$ & $<0.00 \mathrm{I}^{\mathrm{a}}$ \\
\hline \multicolumn{4}{|l|}{ History of } \\
\hline Diabetes mellitus & $19(9.6 \%)$ & $12(3.2 \%)$ & $0.004^{b}$ \\
\hline Hypertension & $64(32.3 \%)$ & 97 (27.4\%) & $0.387^{b}$ \\
\hline Albumin $(g / d L)$ & $4.4 \pm 0.3$ & $4.4 \pm 0.3$ & $0.614^{\mathrm{a}}$ \\
\hline \multicolumn{4}{|l|}{ Smoking status } \\
\hline No & $72(36.4 \%)$ & $293(82.8 \%)$ & $<0.00 \mathrm{I}^{\mathrm{b}}$ \\
\hline Current & $52(26.3 \%)$ & $23(6.5 \%)$ & \\
\hline Previous & 74 (37.4\%) & 38 (10.7\%) & \\
\hline \multicolumn{4}{|l|}{ Habitual alcohol intake } \\
\hline No & $42(21.2 \%)$ & 245 (69.2\%) & $<0.00 \mathrm{I}^{\mathrm{b}}$ \\
\hline Current & I 48 (74.7\%) & 97 (27.4\%) & \\
\hline Previous & $8(4.0 \%)$ & $12(3.4 \%)$ & \\
\hline \multicolumn{4}{|l|}{ Marital status } \\
\hline Married & $175(88.4 \%)$ & $264(74.6 \%)$ & $<0.00 \mathrm{I}^{\mathrm{b}}$ \\
\hline Unmarried & $23(11.6 \%)$ & 90 (25.4\%) & \\
\hline \multicolumn{4}{|l|}{ Level of education } \\
\hline 9 years & $52(26.3 \%)$ & 78 (22.0\%) & $<0.00 \mathrm{I}^{\mathrm{b}}$ \\
\hline 12 years & $115(58.1 \%)$ & $192(54.2 \%)$ & \\
\hline 14 years & $16(8.1 \%)$ & $72(20.3 \%)$ & \\
\hline 16 years & $15(7.6 \%)$ & $12(3.4 \%)$ & \\
\hline \multicolumn{4}{|l|}{ Exercise habit } \\
\hline None & I 32 (66.7\%) & $216(61.0 \%)$ & $0.227^{b}$ \\
\hline I day/week & 22 (II.I\%) & 33 (9.3\%) & \\
\hline 2-3 days/week & $17(8.6 \%)$ & 39 (11.0\%) & \\
\hline 4-5 days/week & $13(6.6 \%)$ & $21(5.9 \%)$ & \\
\hline $\begin{array}{l}6 \text { days/week or } \\
\text { every day }\end{array}$ & 14 (7.1\%) & 45 (I2.7\%) & \\
\hline Nonmenopause & & 73 (20.6\%) & \\
\hline Menopause & & $28 \mid(79.4 \%)$ & \\
\hline
\end{tabular}

Notes: aKruskal-Wallis test; ${ }^{b}$ chi-squared test. Values are presented as mean $\pm S D$, or $\mathrm{n}(\%)$.

Abbreviations: BMI, body mass index; SD, standard deviation; SMM, skeletal muscle mass.

confounding factors, the number of teeth was shown to be an independent risk factor for the timed $10 \mathrm{~m}$ walk test (in females) and SMM (in males).

\section{Relationship between the dental occlusion and the muscle mass and its function}

Table 3 shows the results of the analysis of covariance with adjustment for confounding factors and reveals that the timed $10 \mathrm{~m}$ walk test was significantly correlated with the Eichner 
Table 2 Multiple regression analysis showing associations between the number of teeth and the muscle mass and its function in males

\begin{tabular}{|c|c|c|c|c|c|c|}
\hline & \multicolumn{6}{|c|}{ Dependent variables } \\
\hline & \multicolumn{2}{|c|}{$\begin{array}{l}\text { Timed } 10 \mathrm{~m} \\
\text { walk test (s) }\end{array}$} & \multicolumn{2}{|c|}{$\begin{array}{l}\text { Hand grip } \\
\text { strength (kg) }\end{array}$} & \multicolumn{2}{|c|}{ SMM (kg) } \\
\hline & $\bar{\beta}$ & $P$-value & $\bar{\beta}$ & $\overline{P \text {-value }}$ & $\bar{\beta}$ & $P$-value \\
\hline \multicolumn{7}{|c|}{ Number of teeth } \\
\hline Males & -0.128 & 0.088 & 0.092 & 0.198 & 0.111 & 0.031 \\
\hline Females & -0.157 & 0.007 & -0.086 & 0.145 & -0.045 & 0.400 \\
\hline
\end{tabular}

Notes: Adjusted for BMI, diabetes, hypertension, serum albumin concentration $(\mathrm{g} / \mathrm{dL})$, smoking status, habitual alcohol intake, marital status (living vs not living with a spouse), level of education, and exercising habits. $\beta$ denotes the standardized partial regression coefficients.

Abbreviations: BMI, body mass index; SMM, skeletal muscle mass.

index (Classes $\mathrm{A}$ and $\mathrm{C}$ in females were correlated). Both hand grip strength and SMM were not significantly correlated with dental occlusion.

\section{Discussion}

The present cross-sectional study investigated the occlusal condition and its correlation with physical condition and muscle mass in a community-dwelling Japanese population. Because the participants in this study lived in the Tohoku region, most of them were engaged in agriculture. Physical fitness of individuals in the rural area may be higher than that of individuals in the urban area. In females, the number of teeth present showed a negative correlation with the timed $10 \mathrm{~m}$ walk test, and the timed $10 \mathrm{~m}$ walk of females in Class A was faster than those in Class C. Thus, decline in the number of teeth and occlusal support region may be the risk factors for lower speed in the timed $10 \mathrm{~m}$ walk test. This mechanism can be explained by the decreased mastication due to the limited number of teeth, resulting in reduced

Table 3 Analysis of covariance results showing associations between the Eichner index and the muscle mass and its function

\begin{tabular}{|c|c|c|c|}
\hline $\begin{array}{l}\text { Eichner } \\
\text { index }\end{array}$ & $\begin{array}{l}\text { Timed } 10 \mathrm{~m} \\
\text { walk test (s) }\end{array}$ & $\begin{array}{l}\text { Hand grip } \\
\text { strength (kg) }\end{array}$ & SMM (kg) \\
\hline \multicolumn{4}{|l|}{ Males } \\
\hline Class A & $4.4 \pm 0.1$ & $41.3 \pm 0.6$ & $49.5 \pm 0.3$ \\
\hline Class B & $4.4 \pm 0.2$ & $39.5 \pm 1.4$ & $49.3 \pm 0.7$ \\
\hline Class C & $4.5 \pm 0.2$ & $41.0 \pm 1.5$ & $48.2 \pm 0.8$ \\
\hline \multicolumn{4}{|l|}{ Females } \\
\hline Class A & $4.6 \pm 0.5$ & $25.4 \pm 0.2$ & $35.4 \pm 0.2$ \\
\hline Class B & $4.7 \pm 0.1\} *$ & $25.2 \pm 0.5$ & $35.5 \pm 0.3$ \\
\hline Class C & $5.0 \pm 0.1$ & $26.1 \pm 0.5$ & $35.6 \pm 0.4$ \\
\hline
\end{tabular}

Notes: Data presented as mean \pm standard deviation. Adjusted for age, BMI, diabetes, hypertension, serum albumin concentration $(\mathrm{g} / \mathrm{dL})$, smoking status, habitual alcohol intake, marital status (living vs not living with a spouse), level of education, and exercise habit. *Class $A$ and Class $C$ in females were correlated $(P<0.05)$.

Abbreviations: BMI, body mass index; SMM, skeletal muscle mass. stimulation of the nerve center through the periodontal membrane that caused a reduction in physical functions such as ambulatory speed. Kawakubo et al assessed the effect of teeth clenching on the force of hand grip and cortical activity during the occurrence of facilitatory effects using functional magnetic resonance imaging. ${ }^{17}$ They suggested that occlusal function may affect the function of remote muscles through cortical activation, which supported the findings listed previously in this study. In contrast, when ambulatory speed is slower, physical function and muscle mass may decrease, causing poor oral hygiene, resulting in loss of teeth.

Lower muscle mass is considered to influence QOL because it is associated with increased risk of mobility loss. According to the EWGSOP, Buchner et al showed that small changes in physiological capacity may have substantial effects on physical performance in frail adults, while large changes in physiological capacity have little or no effect in healthy adults. ${ }^{18,19}$ Some researchers have suggested that timed usual gait provides a predictive value for the onset of disability, severe mobility limitation, and mortality. ${ }^{20,21}$ Another study reported that lifestyles of elderly persons with $\geq 20$ remaining teeth tend to be more active than those with $<20$ remaining teeth, which supports the findings of this study. ${ }^{22}$ Considering the care of the elderly and frailty of daily life, timed usual gait is more important than muscular strength. This study showed that the number of teeth and occlusal condition were related to the results of the timed $10 \mathrm{~m}$ walk test. Therefore, worsened mastication function may be a predictor of frailty or severe movement restrictions and even death.

In contrast to females, the number of teeth did not show significant correlations with the timed $10 \mathrm{~m}$ walk in males $(P=0.088)$, whereas it showed positive correlation with total muscle mass. Poor mastication function caused by loss of teeth and limited occlusal support region influence the amount of food intake and its quality. In particular, in case of periodontal disease, chronic inflammation, producing inflammatory cytokines, results in loss of muscle mass throughout the body. ${ }^{23-26}$ Loss of muscle mass affects certain physical functions (eg, brushing teeth and gargling) that are associated with maintaining dental hygiene, which may worsen the periodontal diseases.

Furthermore, a previous epidemiological study reported that in persons aged 40-79 years, the total SMM decreased by $10.8 \%$ in males and $6.4 \%$ in females; the percentage of the total SMM was greater in males than in females. ${ }^{9}$ Furthermore, Tanimoto et al investigated the influence of age on muscle mass by estimating the muscle mass of the upper 
and lower limbs, trunk, and whole body in 4,003 communitydwelling Japanese elderly and found that muscle mass of the lower limbs was the first to be affected and showed the greatest decrease. ${ }^{27}$ However, loss of muscle due to aging needs to approach a certain level to affect the ambulatory speed of a person. ${ }^{28}$ Thus, reduced muscle in lower limb in males was not considered to have affected the ambulatory speed in this study, mainly due to the presence of greater muscle mass in males than in females. In brief, they have enough muscle in the lower limb to overpower the high decrease rate of muscle mass with age in males, resulting in no apparent changes in their walking.

For both females and males, decreased number of teeth caused reduced total muscle mass and ambulatory speed and suggested lower QOL. However, preventing teeth loss may be more important in females because they showed greater decrease in ambulatory speed, which is closely associated with QOL.

\section{Limitations}

There were several limitations in this study that warrant mention. First, the study design was cross-sectional, which did not enable identifying the detailed causal connections among the measured items. Thus, further research with a longitudinal design is required. Second, the total SMM measurement was estimated using bioelectrical impedance analysis in this study, which was not the method recommended by the EWGSOP for its assessment. Third, several potential confounding factors, such as socioeconomic level and use of dentures, were not assessed in this study. This was largely due to the fact that the socioeconomic statuses of individuals are considered a part of personal information in Japan, and thus, we were unable to include it in this study. Fourth, all participants in this study were volunteers who showed interest in their own health, who may have been healthier than other local residents. In brief, the muscle mass and its function and the number of teeth of those who did not participate in this study may differ from the subjects of this study. This selection bias must also be considered in studies targeting community populations. Finally, due to our relatively small sample size, we were unable to exclude beta error as the cause of our failure to detect associations between the number of teeth and the muscle mass and its functions.

\section{Conclusion}

The authors believe that prevention of teeth loss is important for maintaining muscle strength and its function in people aged 40-79, especially for walking ability. This cross-sectional study on a Japanese community-dwelling population revealed relationships between partial oral conditions and the muscle mass and its function. However, the interpretation of our results was hampered by a lack of data, including those on socioeconomic status and longitudinal observations. Therefore, future research exploring teeth loss and the muscle mass and its function is warranted.

\section{Acknowledgments}

The authors thank all their coworkers in this study (especially Youhei Sekine, Daisuke Chiba, Satou Satoshi, Rina Tanaka, Hiroshi Ishizaki, Takao Noguchi) for skillful contributions to the collection and management of the data. This study was supported by grants-in-aid for Scientific Research (15K20847) from the Japanese Ministry of Education, Culture, Sports, Science, and Technology and research aid from the Foundation Institute of Geriatric Medicine and Dentistry in Tokyo.

\section{Author contributions}

AI conceived the study and wrote the initial draft of the manuscript. IT designed the study, conducted the statistical analyses, interpreted the data, and assisted in drafting the manuscript. KS contributed to the study design and interpretation of the results and had full access to all the data in the study. IT and KS participated in the data collection. WK took responsibility for the integrity of the data and the accuracy of the data analysis. All authors have read and approved the final manuscript. All authors contributed toward data analysis, drafting and revising the paper and agree to be accountable for all aspects of the work.

\section{Disclosure}

The authors report no conflicts of interest in this work.

\section{References}

1. Moriya S, Muramatsu T, Tei K, et al. Relationships between oral conditions and physical performance in a rural elderly population in Japan. Int Dent J. 2009;59:369-375.

2. Takata Y, Ansai T, Awano S, et al. Relationship of physical fitness to chewing in an 80-year-old population. Oral Dis. 2004;10:44-49.

3. Budtz-Jorgensen E, Chung JP, Rapin CH. Nutrition and oral health. Best Pract Res Clin Gastroenterol. 2001;15:885-896.

4. Sahyoun NR, Lin CL, Krall E. Nutritional status of the older adult is associated with dentition status. J Am Diet Assoc. 2003;103:61-66.

5. Österberg T, Mellstrom D, Sundh V. Dental health and functional aging. A study of 70-year-old people. Community Dent Oral Epidemiol. 1990;18:313-318.

6. Yamaga T, Yoshihara A, Ando Y, et al. Relationship between dental occlusion and physical fitness in an elderly population. J Gerontol A Biol Sci Med Sci. 2002;57:M616-M620.

7. Delmonico MJ, Harris TB, Lee JS, et al. Alternative definitions of sarcopenia, lower extremity performance, and functional impairment with aging in older men and women. J Am Geriatr Soc. 2007;55:769-774. 
8. Goodpaster BH, Park SW, Harris TB, et al. The loss of skeletal muscle strength, mass, and quality in older adults: the health, aging and body composition study. J Gerontol A Biol Sci Med Sci. 2006;61: 1059-1064.

9. Yamada M, Moriguch Y, Mitani T, Aoyama T, Arai H. Age-dependent changes in skeletal muscle mass and visceral fat area in Japanese adults from 40 to 79 years of age. Geriatr Gerontol Int. 2014;14(S1):8-14.

10. Akune T, Muraki S, Oka H, et al. Exercise habits during middle age are associated with lower prevalence of sarcopenia: the ROAD study. Osteoporos Int. 2014;25:1081-1088.

11. Nishiguchi S, Yamada M, Kajiwara Y, et al. Effect of physical activity at midlife on skeletal muscle mass in old age in community-dwelling older women: A cross-sectional study. J Clin Gerontol Geriatr. 2014;5:18-22.

12. Ministry of Health Labour and Welfare. Statistical tables of the survey of dental diseases. Available from: http://www.mhlw.go.jp. Accessed June 1, 2016.

13. Eichner K. Über eine Gruppeneinteilung des Lückengebisses für die Prothetik. Dtsche Zahnärztl Z. 1955;10:1831-1834.

14. Wolf S L, Catlin PA, Gage K. Establishing the reliability and validity of measurements of walking time using the Emory Functional Ambulation Profile. Phys Ther. 1999;79(12):1122-1133.

15. Bohannon RW, Andrews AW, Thomas MW. Walking speed: reference values and correlates for older adults. J Orthop Sports Phys Ther. 1996; 24:86-90.

16. Wade DT, Wood VA, Heller A, Maggs J, Langton Hewer R. Walking after stroke: measurement and recovery over the first 3 months. Scand J Rehabil Med. 1987;19:25-30.

17. Kawakubo N, Miyamoto JJ, Katsuyama N, et al. Effects of cortical activations on enhancement of handgrip force during teeth clenching: an fMRI study. Neurosci Res. 2014;79:67-75.

18. Cruz-Jentoft AJ, Baeyens JP, Bauer JM, et al. Sarcopenia: European consensus on definition and diagnosis report of the European Working Group on Sarcopenia in older people. Age Ageing. 2010;39:412-423.
19. Buchner DM, Larson EB, Wagner EH, Koepsell TD, de Lateur BK. Evidence for a non-linear relationship between leg strength and gait speed. Age Ageing. 1996;25:386-391.

20. Guralnik JM, Ferrucci L, Pieper CF, et al. Lower extremity function and subsequent disability: consistency across studies, predictive models, and value of gait speed alone compared with the short physical performance battery. J Gerontol A Biol Sci Med Sci. 2000;55:M221-M231.

21. Cesari M, Kritchevsky SB, Newman AB, et al. Added value of physical performance measures in predicting adverse health-related events: results from the health, aging and body composition study. J Am Geriatr Soc. 2009;57:251-259.

22. Tada A, Watanabe T, Yokoe H, Hanada N, Tanzawa H. Relationship between the number of remaining teeth and physical activity in community-dwelling elderly. Arch Gerontol Geriatr. 2003;37:109-117.

23. Yoshihara A, Watanabe R, Nishimuta M, Hanada N, Miyazaki H. The relationship between dietary intake and the number of teeth in elderly Japanese subjects. Gerodontology. 2005;22:211-218.

24. Bretz WA, Weyant RJ, Corby PM, et al. Systemic inflammatory markers, periodontal diseases, and periodontal infections in an elderly population. J Am Geriatr Soc. 2005;53:1532-1537.

25. Sun XJ, Meng HX, Shi D, et al. Elevation of C-reactive protein and interleukin-6 in plasma of patients with aggressive periodontitis. J Periodontal Res. 2009;44:311-316.

26. Schaap LA, Pluijm SM, Deeg DJ, Visser M. Inflammatory markers and loss of muscle mass (sarcopenia) and strength. Am J Med. 2006; 119:526:e9-e17.

27. Tanimoto Y, Watanabe M, Kono R, Hirota C, Takasaki K, Kono K. Aging changes in muscle mass of Japanese. Nihon Ronen Igakkai zasshi. 2009;47:52-57. [Japanese.]

28. Buchner DM, Larson EL, Wagner EH, Koepsell TD, de Lateur BJ. Evidence for non-linear relationship between leg strength and gait speed. Age Ageing. 1996;24:386-391.
Clinical Interventions in Aging

\section{Publish your work in this journal}

Clinical Interventions in Aging is an international, peer-reviewed journal focusing on evidence-based reports on the value or lack thereof of treatments intended to prevent or delay the onset of maladaptive correlates of aging in human beings. This journal is indexed on PubMed Central, MedLine,

\section{Dovepress}

CAS, Scopus and the Elsevier Bibliographic databases. The manuscript management system is completely online and includes a very quick and fair peer-review system, which is all easy to use. Visit http://www.dovepress. com/testimonials.php to read real quotes from published authors. 